science" (182), claims that the history of physiognomy and the development of its ancient beliefs into racist ones was not one-way. The author maintains that symbols of racist prejudice did not appear as such in texts of learned physiognomy, because they were "so individualistic that questions of parental transmission of traits and hereditary were relegated to a marginal position" (199).

In conclusion, this collection of essays seems to be the proper completion of Isaac's volume The Invention of Racism in Classical Antiquity, because it plugs its significant lacunae, providing studies on topics that are rarely treated by modern historical scholarship.

SCILTIAN GASTALDi, University of Toronto

Furno, Martine (dir.).

Qui écrit ? Figures de l'auteur et des co-élaborateurs du texte. $X V^{e}-X V I I I^{e}$ siècle. Collection " Métamorphoses du livre ». Lyon: ENS Éditions / Institut d'histoire du livre, 2009. 262 p. ISBN 978-2-84788-178-3 (broché) $19 €$

On a claironné jadis la mort de l'auteur. Des sirènes - électroniques — chantent maintenant celle du livre (imprimé). Mais si on s'était trompé quant à notre conception même de la figure de l'auteur comme de la notion de l'objet-livre ? Si ces morts en devenir n'avaient jamais été que des cristallisations récentes et historiquement passagères associées à ce que certains, à la suite du chercheur danois L. O. Sauerberg, ont appelé la "parenthèse Gutenberg », serait-on autorisé à en dramatiser ainsi la date de péremption ?

Ce recueil s'interroge justement sur la nature polymorphe des textes et des auteurs qui sont à la source des livres dans l'Ancien Régime - au XVI siècle tout particulièrement (la périodisation $\mathrm{XV}^{\mathrm{e}}-\mathrm{XVIII}{ }^{\mathrm{e}}$ annoncée en page couverture est un peu trompeuse), alors donc que s'ouvre cette parenthèse Gutenberg. "Qui écrit ? » demande-t-on. Le sous-titre, plus modeste, apporte déjà un élément de réponse en proposant de redistribuer le pouvoir auctorial entre la " figure » de l'auteur et celle des «co-élaborateurs » du texte, terme englobant par lequel les auteurs du volume désignent les éditeurs, traducteurs, 
commentateurs, imprimeurs et autres intervenants qui participent à divers titres et degrés à la production des œuvres manuscrites ou imprimées.

Le livre, issu d'un colloque tenu à Grenoble en 2006, est lui-même le résultat d'une co-élaboration intellectuelle, institutionnelle et éditoriale qui aurait pu donner naissance à un ouvrage hétérogène (typique des actes de colloques), si ce n'avait été d'un louable effort de « mise en perspective des questions scientifiques » qui accorde une cohérence certaine à l'ensemble, même si certaines interventions, un peu cantonnées dans leur érudition, ne répondent pas aux ambitions affichées dans le titre de l'ouvrage.

Dans son introduction, brève mais pertinente, Martine Furno précise que les études réunies tentent d'approcher la figure de l'auteur non pas sous l'angle de l'auctorialité mais sous celui de l'autorité, à une époque et dans des situations où cette autorité est encore "mal fixée ou multiforme ", et ce, en s'intéressant tout particulièrement aux «mains multiples » du livre. L'introduction est relayée par de courtes présentations - témoignant d'un réel effort éditorial - au seuil des quatre chapitres dans lesquelles sont regroupées les douze contributions.

Dans le premier chapitre sur les entreprises collectives, hormis la contribution intempestive du dix-huitiémiste de Benoît Gain qu'on ne commentera pas ici, on trouve des interventions d'Isabelle Diu et de Raphaële Mouren. La première s'intéresse à l'exemple révélateur de la traduction patristique dans le cercle d'Érasme dont la situation paradoxale en tant qu'auteur se voit redoublée dans l'entreprise de traduction où ce " passeur de l'Autre » se fait aussi auteur avec le concours d'un vaste réseau de collaborateurs. L'exemple des traductions de Saint-Chrysostome permet de mettre en relief l'importance du modèle humaniste du dialogue entre auteur, traducteurs et collaborateurs de ces grands chantiers de publication typiques de la nouvelle république des lettres. Le travail d'équipe sur les éditions grecques du milieu du XVI ${ }^{e}$ siècle permet ensuite à Raphaële Mouren (à partir surtout d'exemples liés à Piero Vettori) d'illustrer, avec rigueur, la nature des « interventions » nécessaires dans le processus de production et d'impression de ces ouvrages qui impliquent une « organisation de travail de groupe ». Ainsi, avec Vettori, plusieurs collaborateurs et un véritable « atelier de travail » contribuent aux diverses étapes de l'édition scientifique, qui se voit aussi nourrie, ici encore, par les dialogues et polémiques avec d'autres humanistes.

Le chapitre suivant pose la question des " contraintes de l'impression " à partir de deux cas significatifs d'auteurs ayant manqué leur " rendez-vous » 
avec l'imprimerie. Laurence Pradelle s'attarde d'abord au destin auctorial de Leonardo Bruni, décédé avant l'invention (européenne) de la presse à imprimer, mais qui n'en avait pas moins atteint un statut d'auteur tel qu'on lui a maintes fois attribué des œuvres dont il n'était pas l'auteur. Pradelle s'intéresse à divers cas de figure, mais s'arrête surtout au cas du Disputatio de nobilitate attribué de manière aussi fréquente à Bruni qu'à Buonaccorso da Montemagno. Selon Pradelle, le fait qu'il s'agirait d'un pastiche polémique inspiré du style de Bruni pourrait avoir confondu des copistes (dont certains vont jusqu'à modifier le texte pour le rendre plus conforme à Bruni !), ce qui témoigne du caractère alors ambigu de la notion d'auteur dont la part d'autorité entretient des liens très variables avec sa part d'authenticité. Jean-Eudes Girot, quant à lui, étudie le cas tout à fait significatif du poète Mellin de Saint-Gelais qui, écrivant à l'époque où l'imprimerie est en plein essor, refuse de faire appel au nouveau médium. Girot montre bien comment le contrôle de la diffusion, rendu possible par le travail de sélection et d'édition qu'impose l'impression, est crucial pour la constitution de la figure de l'auteur. Ayant choisi de privilégier la noble vie de poète de cour plutôt que de se soumettre aux pratiques des imprimeurs et de l'institution littéraire naissante, ce "poète éparpillé » verra donc l'autorité même de ses œuvres diminuée tant aux yeux de ses contemporains que de la postérité.

Le troisième chapitre montre comment des enjeux semblables ont cours dans les milieux moins étudiés des publications scolaires et techniques, domaines où les co-élaborateurs sont souvent condamnés à un oubli rapide. Les deux premières contributions concernent des écrits liés à l'apprentissage du latin dans des contextes très différents. Nathaël Istasse présente avec soin les rouages de la rédaction, de l'édition, de la correction et de la diffusion d'ouvrages didactiques de l'humaniste Nivernais, célèbre en son temps, Johannes Ravisius Textor, plus particulièrement son Epitheta et son Officina, ouvrages qui - au fil de leurs innombrables éditions - font l'objet de nombreuses révisions et transformations sous l'influence du compilateur lui-même, de ses éditeurs et de son public cible. Joaquín Pascual Barea nous entraîne ensuite dans l'univers de la poésie latine et néolatine sévillane afin de mettre en lumière le rôle, indirect mais très influent, que peuvent jouer des « co-élaborateurs " n'ayant aucunement mis la main à l'ouvrage. En effet, l'identité de l'archevêque qui nommait l'écolâtre de l'Estudio San Miguel semble avoir joué un rôle majeur dans l'orientation de cette poésie : selon que l'archiépiscopat ait été assuré par un disciple de Nebrija, un inquisiteur plus sévère ou un érasmien, la 
nature même de la poésie et des ouvrages publiés s'en est vu profondément transformée. Les deux contributions suivantes nous transportent dans l'univers des traités d'architecture. À partir de l'exemple des éditions, traductions, abrégés et annotations du De architectura de Vitruve, Frédérique Lemerle met en relief l'importance des interférences induites par l'intervention d'éditeurs scientifiques, de traducteurs, de commentateurs et d'illustrateurs aux objectifs parfois divergents. Des éditions « composites» - telle la traduction française de Jean Martin (1547) accompagnée des illustrations du sculpteur Jean Goujon permettent de comprendre comment les éditions, traductions et annotations du traité vitruvien révèlent non seulement la culture de leurs auteurs et collaborateurs, mais aussi leur propre conception de l'architecture, ce qu'Yves Pauwels confirme en montrant ensuite la nature tout aussi composite des éditions de la Reigle generale... de Jean Bullant (publiée pour la première fois à Paris en 1564), ouvrage qui, tant dans ses textes que ses images doit finalement assez peu à son auteur putatif, l'architecte du château d'Écouen.

Le dernier chapitre de l'ouvrage, le plus intéressant selon moi, s'intéresse aux imprimeurs qui publient « leurs propres textes " à partir d'exemples dus à deux des plus éminents représentants de la dynastie des Estienne. Chantal Liaroutzsos effectue d'abord un relevé chronologique attentif des œuvres de Charles Estienne qui lui permet de proposer une lecture de la «fonction auteur » qui interroge, comme c' est trop peu souvent le cas dans cet ouvrage, des hypothèses théoriques (Foucault ici). Les exemples étudiés montrent une forme de gradation du statut d'auteur : quasi nul dans les vocabulaires et abrégés que publie Estienne en tant que compilateur; " plus digne d'être mentionné » quand il se fait traducteur ; enfin, beaucoup plus affirmé dans le De dissectione (1545) pour lequel son auteur peut revendiquer une indéniable autorité (pratique). Cependant, le cas de l'œuvre foncièrement "plurielle " qu'est Le guide des chemins de France (1552), où Estienne revient au quasi-anonymat, montre que sa conception de la fonction d'auteur n'est pas que liée à son autorité, mais aussi à sa «fonction de transmission », ce qui met en lumière l'importante relation entre théorie et pratique chez les humanistes (ainsi qu'entre les « mots et les choses "). Martine Furno souhaite, quant à elle, démontrer l'intérêt des pièces liminaires des imprimeurs en se penchant sur l'exemple "extrême » des deux préfaces que rédige Robert pour son édition des Forensia de Budé (1544), puis pour le volume correspondant des Indices (1545). Même s' il demeure impossible — et anachronique, admet-elle - de tenter de déterminer « exactement» la part 
que Robert Estienne a contribué à ces ouvrages — où il se présente soit comme « exécutant technique » et « imprimeur scrupuleux » ou, dans le deuxième cas, comme une sorte de "logisticien du volume » -, on peut reconnaître la main de l'imprimeur à tout le moins dans le classement et la structure des Indices à tout le moins, si bien, qu'il faudrait voir Estienne comme auteur « secondaire ou associé » de cet ouvrage. Le fait qu'Estienne se cache ainsi humblement derrière des autorités "plus ou moins fictives » dans la majorité de ses publications à l'exception de ses éditions de la Bible - s'expliquerait notamment par des raisons commerciales, tandis que, dans le domaine des lettres sacrées, Estienne souhaiterait être fidèle à sa foi. Dans la dernière contribution de ce recueil, Hélène Cazes explore le « paradoxe de l'éditeur » à partir de l'exemple probant des Censures... que publie justement en 1552 ce même Robert Estienne en latin, puis en français à partir de son exil genevois. Ces ouvrages qui reproduisent in extenso les condamnations de la Faculté de théologie de Paris paraissent exprimer " un refus de la position d'auteur ", mais Cazes dissèque habilement la «fière humilité » derrière laquelle se réfugie Estienne qui, par cette stratégie argumentative de l'effacement, institue un procès, en prenant les lecteurs pour témoins, aux institutions qui le "martyrisent». Le fait que la principale pièce à conviction de ce procès éditorial soit son édition de la Bible lui permet de jouer sur deux tableaux : celui du procès qu' on lui intente et celui, plus important, des Écritures qu'il ne souhaiterait que «donner à lire ». Ainsi, l'éditeur-imprimeur devient auteur à travers son silence, en écrivant en quelque sorte "sous la dictée du sainct Esprit », ultime co-élaborateur du livre.

Ce livre collectif n'a sans doute pas pu bénéficier d'un tel patronage spirituel, mais on voit bien qu'il a été préparé avec soin par des " gens du livre » : il contient une bibliographie générale, une "Liste des éditions citées antérieures à 1800 ", une "Liste des manuscrits cités ", ainsi qu'un index assez complet. Le volume me parait d'ailleurs témoigner du « tournant matériel » relativement récent dans certaines disciplines des humanités de plus en plus intéressées par la matérialité du livre, son processus de production, son contexte de publication, etc.

Serait-ce le «tournant numérique » contemporain qui aurait rendu le médium du livre visible au-delà des disciplines traditionnellement liées à la bibliographie matérielle ? Si c'est bien le cas, il serait intéressant de poursuivre la réflexion et de se demander jusqu'à quel point certains aspects transitionnels de cette période de gestation qu'est la Renaissance peuvent être utiles pour comprendre notre propre transition médiatique. Les modalités des rapports 
entre auteurs, éditeurs et autres «co-élaborateurs » des nouveaux documents électroniques ne sont-elles pas aussi en pleine négociation, en voie d'évoluer vers des modes d'opération qui ne se sont pas encore cristallisés ? "Qui écrit » aujourd'hui sur le Web, les blogues, sur les réseaux sociaux, ainsi que dans le monde en plein essor de l'édition électronique, alors que la parenthèse Gutenberg semble être en voie de se refermer? Des études rigoureuses comme celles-ci sur le moment où s'ouvrait cette même parenthèse pourraient certainement apporter une contribution significative à la réflexion.

jean-françois vallée, Collège de Maisonneuve

\section{Gavitt, Philip.}

Gender, Honor, and Charity in Late Renaissance Florence.

New York: Cambridge University Press, 2011. Pp. x, 280. ISBN 978-1-10700294-4 (hardback) \$90.

In this engaging new study, Philip Gavitt returns to issues raised in his previous work on charity, children, and family in Renaissance Florence. Taking up the question of families, Gavitt seeks to explain the abandonment crisis and proliferation of new charitable institutions dedicated to women and girls in late sixteenth-century Florence. Blending social, religious, institutional, political, and gender history Gavitt offers a carefully constructed picture of the challenges faced by Florentine families, the Medici government, and the city's hospitals and convents as inheritance laws clashed with social realities. Gavitt argues that the rise in child abandonment coincided with the development of a status culture of consumption and that families, in order to preserve their honour and patrimonies, had to work within this status culture while still providing for children who fell outside the lines of inheritance. Viewed in this light, charitable institutions played a crucial role in the lineage strategies of Florentine families of all social classes.

Before discussing charitable institutions and lineage strategies, Gavitt first addresses various contexts, including the social and economic crises of the sixteenth century, Medici government reforms and state-building, and late Renaissance ideologies of discipline and civility. These contexts established, 